\title{
Corrigendum: Brain feminization requires active repression of masculinization via DNA methylation
}

Bridget M Nugent, Christopher L Wright, Amol C Shetty, Georgia E Hodes, Kathryn M Lenz, Anup Mahurkar, Scott J Russo, Scott E Devine \& Margaret M McCarthy

Nat. Neurosci. 18, 690-697 (2015); published online 30 March 2015; corrected after print 8 February 2017

In the version of this article initially published, the Whole Genome Bisulfite Sequencing (WGBS) analysis was based on a data set in which one of the female .bam alignment files (FV2) was mistakenly truncated. This file has been removed from NCBI and updated with the raw reads. The realigned raw sequencing reads yielded the same alignment statistics as originally reported in Supplementary Table 5 . The reanalysis did not change the main finding that females have significantly more fully methylated CpG sites in the POA than males and estradiol-treated females. In both analyses the reads were filtered for coverage, with a minimum of three reads per site per sample required for inclusion in the analysis. Thus reanalysis with the new, untruncated FV2 file meant that additional CpG and CHG sites became available for analysis across all samples. The analysis of this expanded data set showed a slightly different distribution of sex differences in CpG methylation across genomic regions (Fig. 1d) and chromosomes (Supplementary Fig. 3) than previously reported. After reanalysis incorporating the missing data, Figure 1c,d and Supplementary Figures 2 and 3 have been replaced. In the first Results paragraph, "females had nearly twice the level of fully (100\%) methylated CpG sites as males or masculinized females" has been replaced by "females had nearly 50\% more fully (100\%) methylated CpG sites as males or masculinized females"; "sex differences were generally dispersed across chromosomes, although methylation on chromosome 5 and 13 was biased toward females and males, respectively" has been replaced by "sex differences were generally dispersed across chromosomes"; and "The overwhelming majority of CpG sites exhibiting a sex difference in methylation were in intergenic regions ( $\sim 84 \%)$, followed by introns ( 14\%), promoter regions $(\sim 2 \%)$ and exons $(<1 \%)$ " has been replaced by "The overwhelming majority of CpG sites exhibiting a sex difference in methylation were in intergenic regions $(\sim 69 \%)$, followed by introns $(\sim 26 \%)$, exons $(\sim 5 \%)$ and promoter regions $(<3 \%)$." In the Figure $1 c$ legend, " $F(2,6)=6.594$, $P=0.0306$ " has been replaced by " $F(2,6)=5.67, P=0.041$." In the Figure $1 \mathrm{~d}$ legend, " 1,242 sex differences" has been replaced by " 2,748 sex differences." In the Supplementary Figure 2 legend, “* $p<0.05$ ” has been changed to “** $p<0.01$." Under Supplementary Figure $2 \mathrm{a}$, " $F_{\% m e t h}(9,60)=$ 709.2 " has been changed to " $\mathrm{F}_{\% \text { meth }}(9,60)=1244$ "; " $\mathrm{F}_{\text {sex }}(2,60)=5.047, \mathrm{p}=0.0094$ " has been changed to " $\mathrm{F}_{\text {sex }}(2,60)=2.885, \mathrm{p}=0.0636$ "; "Males and estradiol-treated females had a greater number of CpG sites 80-90\% methylated compared to females (Tukey's HSD, p < 0.0001)" has been changed to "Males (Tukey's HSD, p < 0.0001) and estradiol-treated females (Tukey's HSD, p =0.0014) had a greater number of CpG sites 80-90\% methylated compared to females"; and the final p value has been changed from 0.022 to 0.0055 . Similarly, under Supplementary Figure $2 \mathrm{~b}$, " $F_{\% \text { meth }}(9,60)=4787$ " has been changed to " $F_{\% \text { meth }}(9,60)=8959$ "; " $F_{\text {sex }}(2,60)=7.514, p=0.0012$ " has been changed to " $F_{\text {sex }}(2,60)=12.73$, $\mathrm{p}<0.0001$ "; "male vs. female + e p $=0.0003$ " has been changed to "male vs. female + e p $<0.0001$ "; and " $F_{\text {int }}(18,60)=17.16$ " has been changed to " $\mathrm{F}_{\mathrm{int}}(18,60)=23.8$." In the Supplementary Figure 3 legend, "Overall, male chromosomes were more hypomethylated relative to females $(\chi 2=47.83$, $\mathrm{n}=21, \mathrm{p}=0.0004)$," has been changed to "There were no overall differences in CpG methylation by chromosome $(\chi 2=30.97, \mathrm{n}=20, \mathrm{p}=0.0556)$." The errors have been corrected in the HTML and PDF versions of the article.

Original Figure 1c,d

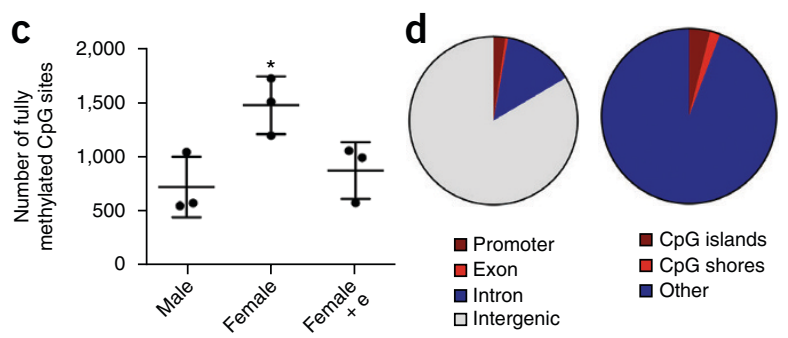

Revised Figure 1c,d

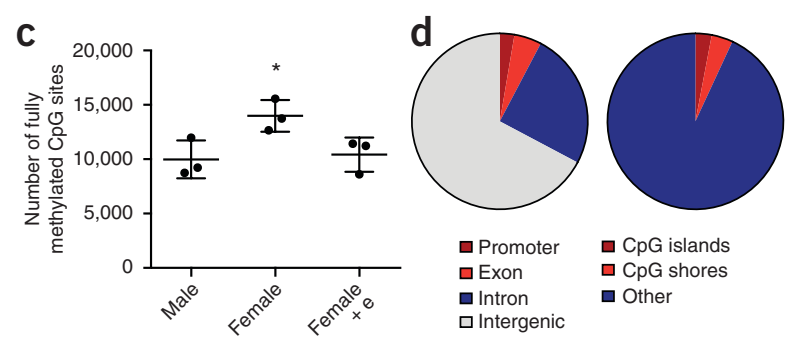

\section{Erratum: Distinct neural mechanisms for the control of thirst and salt appetite in the subfornical organ}

Takashi Matsuda, Takeshi Y Hiyama, Fumio Niimura, Taiji Matsusaka, Akiyoshi Fukamizu, Kenta Kobayashi, Kazuto Kobayashi \& Masaharu Noda

Nat. Neurosci. 20, 230-241 (2017); published online 19 December 2016; corrected after print 31 January 2017

In the PDF and print versions of this article initially published, the year in the online publication date was given as 2017 instead of 2016 . The error has been corrected in the PDF version of the article. 